\title{
Analysis of the Chemo-thermal Stresses around a Spherical Cavity in a Saturated Porous Medium
}

\author{
Pan Xue1, Liang Chen1, Luwu He 1 \\ ${ }^{1}$ School of Mechanical and Power Engineering, East China University of Science and Technology, \\ Shanghai ,200237, China
}

Keywords: Saturated porous medium; Local thermal non-equilibrium; Laplace transform; Pore pressure; Chemo-thermal stress.

\begin{abstract}
In this paper, based on the chemo-thermo-poroelasticity model of saturated porous medium undergoing the local thermal non-equilibrium (LTNE) conditions, the pore pressure and chemo-thermal stresses around a spherical cavity in an infinite fluid saturated porous medium subjected to sudden variations for temperature and solute mole fraction on the cavity boundary are investigated by employing Laplace transform technique and numerical method. The results show that the pore pressure and stresses are dominantly affected by the chemical effect rather than the thermal effect when the temperature and variation of the solute mole fraction change together, and the LTNE effects on radial stress (magnitude) become very pronounced when Sp is small $(\approx 0.35)$ and $\mathrm{Bi}$ is moderate $(\approx 1.0)$. In addition, the pore pressure and stresses are always same order of magnitude whether considering the chemical effect or the chemo-thermal effect.
\end{abstract}

\section{Introduction}

In recent years, the radioactive nuclear waste storage technology has got extensive attention from the researchers. In order to prevent harmful substances in nuclear waste from radiating and penetrating to the surrounding natural environment, radioactive waste (nuclear waste storage tanks, etc.) are often stored in deep underground rock layers (usually in the underground 3000-5000m). Because the physical and chemical reactions in nuclear waste occur frequently and release large amounts of heat, causing the temperature of the surrounding rock or soil barrier body increases, thereby it damages the environment and structure. In engineering, the clay barrier is an important means in solid/fluid waste processing, therefore, the safety assessment of underground rock structure or clay barrier materials under chemical, thermal and mechanical loads has become a very important basic work. In addition, in the process of artificial recharge and underground thermal energy storage (cooled water of steel underground injection), the chemical, thermal and osmotic coupled problems of the soil (typically porous media) also need to be considered. Taking into account the characteristics of the structure and the load, the analysis of the chemo-thermal stresses around a spherical cavity in a saturated porous medium can be simplified to an infinite space spherically symmetric problem. In the local thermal equilibrium (LTE) conditions, there are a number of studies (including consideration of chemical reaction), for example, [1-4].

Minkowycz et al.[5] discussed the local thermal equilibrium (LTE) assumption applicability, and pointed that when the Sparrow number Sp is small, the assumption of the local thermal nonequilibrium (LTNE) conditions need to be adopted (Two energy equation model, namely, in the Representative elementary volume, the solid skeleton and pore fluid have different temperature). For the different combinations of boundary conditions, He and Jin[6,7] use the weighted average temperature to numerical analyze the temperature, pore pressure and thermal stress of the spherical cavity in an infinite fluid saturated poroelasticity under the LTNE conditions, and compare the LTNE effect.

For the pore fluid/solution (consisting of two components for solvent and solute), Ekbote and Abousleiman[8,9] and Ghassemi and Diek[10,11] respectively established a chemo-thermalporoelasticity model of saturated porous medium under the LTE conditions. The difference between two models is that the study objects are different. Based on the weighted average temperature 
concepts[6,7], considering the integrity and consistence of relationship between diffusion flow (flux) and generalized force (driving force), a new chemo-thermal-poroelasticity model of saturated porous medium under the LTNE conditions is established by [12], the chemo-thermal stresses around a spherical cavity in an infinite saturated porous medium are also investigated under the LTE conditions and thermal convection boundary, and the chemical effect and chemo-thermal effect are discussed.

\section{Governing equations}

\subsection{Temperature field}

Assuming isotropic porous elastic media without heat source, under the LTNE conditions, the solid and fluid phase temperatures are governed by the following heat conduction equations [6]

$$
\begin{array}{r}
(1-\phi) \rho_{s} c_{s} \frac{\partial \theta_{s}}{\partial t}-(1-\phi) k_{B z} \Delta \theta_{s}+h_{\text {int }}\left(\theta_{s}-\theta_{f}\right)=0 \\
\phi \rho_{f} c_{f} \frac{\partial \theta_{f}}{\partial t}-\phi k_{\text {Iy }} \Delta \theta_{f}+h_{\text {int }}\left(\theta_{f}-\theta_{s}\right)=0
\end{array}
$$

where $\theta \mathrm{s}$ and $\theta \mathrm{f}$ are the solid and fluid temperature variations respectively, $\mathrm{t}$ is time, $\phi$ is the reference porosity, kTf and kTs is the thermal conductivities of the solid and fluid respectively, $\rho s$ and $\rho f$ are the densities for the solid and fluid respectively, cs and cf are the specific heat for the solid and fluid respectively, hintis the solid-fluid interface heat transfer coefficient, and $\Delta$ is the Laplacian operator. In addition, for the LTE conditions (i.e., temperature of the porous medium $\theta=\theta \mathrm{s}=\theta \mathrm{f}$ ), the fluid-saturated porous medium temperature field can be simplified to the following form.

$$
\frac{\partial \theta}{\partial t}=\kappa \Delta \theta \quad(t>0)
$$

Here $\boldsymbol{\kappa}$ is the thermal diffusivity of fluid saturated porous medium[6,7].

Taking into account the case of the structure and the loads, the analysis of the chemo-thermal stresses around a spherical cavity in an infinite saturated porous medium can be simplified to a spherically symmetric problem, shown as Fig.1. a is hole radius, $r$ is radial coordinate in spherical coordinates. The initial and boundary conditions for the temperatures can thus be formulated as follows:

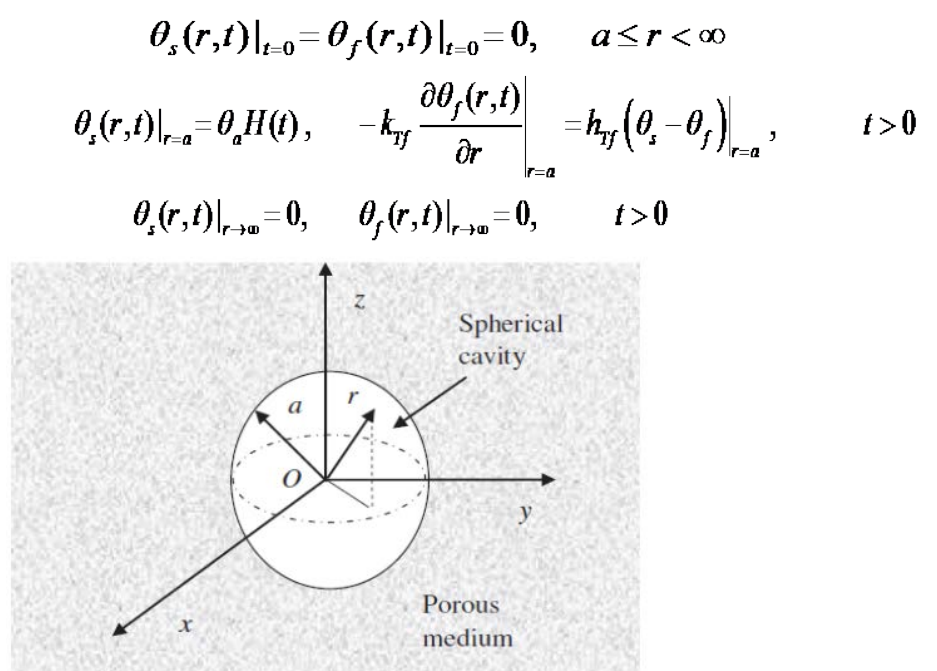

Fig.1 A spherical cavity in an infinite fluid saturated porous medium

where, $\mathrm{H}(\mathrm{t})$ is the Heaviside step function, hTf is the heat transfer coefficient between fluid and nuclear waste storage tanks. When the Biot number $\mathrm{Bi}(=1 / \mathrm{b} 4 \mathrm{f}=\mathrm{a} \mathrm{hTf} / \mathrm{kTf})$ is very large, equation (4) will be degraded to the first boundary condition, that is the temperature of solid phase and flow 
phase at the boundary are given and same.

For the LTE conditions, considering two kinds of the boundary condition, i.e., the convective boundary condition (CBC) and the first boundary condition (PTBC), the initial and boundary conditions for the temperature field can be written as follows:

$$
\begin{array}{r}
\left.\theta(r, t)\right|_{t=0}=0, \quad a \leq r<\infty \\
-\left.k_{T m} \frac{\partial \theta(r, t)}{\partial r}\right|_{r=a}=\left.h_{T M}\left(\theta_{a} H(t)-\theta\right)\right|_{r=a},\left.\quad \theta(r, t)\right|_{r \rightarrow \infty}=0, \quad t>0 \quad(6) \\
\text { or }\left.\quad \theta(r, t)\right|_{r=a}=\theta_{a} H(t),\left.\quad \theta(r, t)\right|_{r \rightarrow \infty}=0, \quad t>0
\end{array}
$$

where $\mathrm{kTm}$ is thermal conductivity of saturated porous media, hTm is heat transfer coefficient between porous media and nuclear waste storage tank.

For the three initial-boundary value problems, (1), (3) and (4); (2), (5) and (6); (2), (5) and (7), the analytical expression of $\theta \mathrm{s}, \theta \mathrm{f}$ and $\theta$ in a Laplace transform domain can be obtained by Laplace transform technique.

\subsection{The chemo-thermal stresses of poroelastic media}

Based on the weighted average temperature concepts[6,7] and the works of Ekbote and Abousleiman[8,9], for the saturated porous medium, in which the pore fluid solution consists of two components (solvent and solute), the governing equations for the displacement, pore pressure and solute molar fraction variation under the LTNE conditions can be written as follows [12]:

$$
\begin{aligned}
& G \Delta\left(u_{i}\right)+\frac{G}{1-2 v} u_{k, k i}-\alpha p_{, i}-K \alpha_{s} \theta_{\text {ave }, i}+\gamma^{s} m_{, i}^{s}=0 \\
& \frac{1}{M} \frac{\partial p}{\partial t}+C_{s} \frac{\partial m^{s}}{\partial t}=B_{p s} \Delta p+B_{m s} \Delta m^{s}+B_{T s} \Delta \theta_{a v e}-\alpha \frac{\partial\left(\varepsilon_{k k}\right)}{\partial t}+\beta^{T s} \frac{\partial \theta_{a v e}}{\partial t} \\
& \frac{1}{M} \frac{\partial p}{\partial t}+C_{f} \frac{\partial m^{s}}{\partial t}=B_{p f} \Delta p+B_{m f} \Delta m^{s}+B_{T f} \Delta \theta_{\text {ave }}-\alpha \frac{\partial\left(\varepsilon_{k k}\right)}{\partial t}+\beta^{T f} \frac{\partial \theta_{\text {ave }}}{\partial t}
\end{aligned}
$$

where ui is the displacement of solid-phase, $\mathrm{p}$ is pore pressure of fluid, $\mathrm{ms}$ is the variation of solute molar fraction, $\varepsilon k k=u k, k, \theta$ ave $=(1-\phi) \theta s+\phi \theta f$.

For an infinite medium under the irrotational displacement field condition, namely ui,j $=u j, \mathrm{i}$, there will be $\Delta u i=\varepsilon k k, i$, and equations (8)-(10) can be simplified as follows:

$$
\begin{aligned}
& \varepsilon_{k k}=\frac{\alpha}{(\lambda+2 G)} p+\frac{K \alpha_{s}}{(\lambda+2 G)} \theta_{\text {ave }}-\frac{\gamma^{s}}{(\lambda+2 G)} m^{s} \\
& \frac{\partial p}{\partial t}=a_{11} \Delta p+a_{12} \Delta m^{s}+a_{13} \Delta \theta_{\text {ave }}+a_{14} \frac{\partial \theta_{\text {ave }}}{\partial t} \\
& \frac{\partial m^{s}}{\partial t}=a_{21} \Delta p+a_{22} \Delta m^{s}+a_{23} \Delta \theta_{\text {ave }}+a_{24} \frac{\partial \theta_{\text {ave }}}{\partial t}
\end{aligned}
$$

here the definition and physical meaning of the coefficients in equations (8)-(13) can refer to the Reference [12]. The above equations (11)-(13) present that the pore pressure, variation of solute molar fraction and the displacements become uncoupled when the displacement field is irrotational in an infinite medium.

For the infinite fluid saturated isotropic porous medium with spherical cavity, shown as Fig.1, assuming that the initial state is the natural state, between the nuclear waste storage tanks and the surrounding soil barrier is non-contact type boundary condition, the pore pressure and the solute molar fraction variation respectively suffer to a sudden increment at the edge of spherical cavity $(\mathrm{r}=$ a), and the solid-phase is free, at this time, the initial and boundary conditions can be expressed as follows:

$$
\begin{gathered}
\left.\sigma_{r}(r, t)\right|_{t=0}=\left.u(r, t)\right|_{t=0}=\left.p(r, t)\right|_{t=0}=\left.m^{s}(r, t)\right|_{t=0}=0, \quad a \leq r<\infty \\
\left.p(r, t)\right|_{r=a}=p_{a} H(t),\left.\quad p(r, t)\right|_{r \rightarrow \infty} \rightarrow 0, \quad t>0
\end{gathered}
$$




$$
\begin{array}{llrl}
\left.m^{s}(r, t)\right|_{r=a}=m_{a}^{s} H(t), & \left.m^{s}(r, t)\right|_{r \rightarrow \infty} \rightarrow 0, & t>0 \\
\left.\sigma_{r}(r, t)\right|_{r=a}=-p_{a} H(t), & \left.\sigma_{r}(r, t)\right|_{r \rightarrow \infty} \rightarrow 0, & t>0
\end{array}
$$

where pa and mas are the sudden increments (constant) of pore pressure and molar fraction of solute respectively, $\mathrm{u}$ is the radial displacement of the solid phase, $\sigma r(r, t)$ is the radial stress of the solid phase.

For the space spherically symmetric problem, the solid phase displacement is irrotational field, therefore, the pore pressure $\mathrm{p}$ and the solute molar fraction ms can be firstly solved by the equations (12) and (13). Combined with the initial and boundary conditions (14)-(16) and the expressions of $\theta \mathrm{s}$ and $\theta \mathrm{f}$ in the Laplace transform domain , the expressions of $\mathrm{p}$ and $\mathrm{ms}$ in Laplace transform domain can be obtained by Laplace transform from equations (12) and (13). After obtaining the temperature filed of solid-phase and fluid-phase $\theta$ s and $\theta f(\theta$ ave $=(1-\phi) \theta s+\phi \theta f)$, and the pore pressure $\mathrm{p}$ and the variation of solute molar fraction $\mathrm{ms}$, the radial displacement $\mathrm{u}$ and the radial stress $\sigma r(r, t)$ and the tangential normal stress $\sigma \varphi(r, t)$ of solid phase can be obtained by the equation of (11) with initial and boundary conditions (14) and (17).

\section{Numerical results}

Since the expressions of temperature field, pore pressure and solute molar fraction in Laplace transform domain are very complicated, the numerical method[13] for the inverse Laplace transform is suitable. In this paper, the clay material as solid phase is considered for numerical computation and analysis, and the specific heat - elastic material parameters (clay) are taken from the References [3,6,7], i.e., $: \phi=0.375, \mathrm{G}=1.2 \mathrm{MPa}, \nu=0.2, \mathrm{~K}=1.6 \mathrm{M} \mathrm{Pa}, \mu=1.0 \times 10-3 \mathrm{~Pa} \cdot \mathrm{s}, \mathrm{K}=5.0 \times 10$ $17 \mathrm{~m} 2, \alpha=1.0, \mathrm{~B}=1.0, \rho \mathrm{s}=2600 \mathrm{~kg} / \mathrm{m} 3, \rho \mathrm{f}=1000 \mathrm{~kg} / \mathrm{m} 3, \mathrm{cs}=937 \mathrm{~J} /(\mathrm{kg} 0 \mathrm{C}), \mathrm{cf}=4200 \mathrm{~J} /(\mathrm{kg} 0 \mathrm{C}), \mathrm{kTs}$ $=3.3 \mathrm{~W} /(\mathrm{m} 0 \mathrm{C}), \mathrm{kTf}=0.6 \mathrm{~W} /(\mathrm{m} 0 \mathrm{C}), \alpha \mathrm{s}=3.0 \times 10-5 / 0 \mathrm{C}, \alpha \mathrm{f}=\alpha \mathrm{s}=3.0 \times 10-4 / 0 \mathrm{C}$. The chemical parameters refer to the References [8-11]: $\gamma^{s}=100 \mathrm{MPa}, \overline{\boldsymbol{m}}^{s}=\mathbf{0 . 1 5}, \overline{\boldsymbol{T}}=\mathbf{3 0 0 \mathrm { K }}, \mathrm{Vs}=3.36 \times 10-5 \mathrm{~m} 3 / \mathrm{mol}$ (Nacl solute), $\mathrm{Vf}=1.8 \times 10-5 \mathrm{~m} 3 / \mathrm{mol}$ (hydrosolvent), $\chi=0.9, \mathrm{R}=8.314 \mathrm{~J} / 0 \mathrm{C}-\mathrm{mol}, \mathrm{Dc}=2.0 \times 10-9 \mathrm{~m} 2 / \mathrm{s}$, DTs $=$ DTf $=1.5 \times 10-9 \mathrm{~m} 2 / \mathrm{s}, \mathrm{a}=0.5 \mathrm{~m}$. The relevant material parameters of isotropic saturated porous media are set as constant, and calculated as follows: $c,=3.72 \times 102 \mathrm{~Pa} / 0 \mathrm{C}, \kappa=4.812 \times 10-7 \mathrm{~m} 2 / \mathrm{s}$, b3 $=0.3553$.

The following mainly discusses the influence of pore pressure and chemo-thermal stress, and LTNE effect and chemical effect. Let $\mathrm{b} 5=\mathrm{kTm} /(\mathrm{ahTm})=\mathrm{b} 4 \mathrm{f}$, and take $\mathrm{pa}=0$ and $\mathrm{h}^{\tilde{}}=\mathrm{a} 2 \mathrm{hint} /(1$ $\phi) \mathrm{b} 3 \mathrm{kTs}=1.0(\mathrm{Sp} \cong \tilde{\mathrm{h}} \mathrm{b} 3=0.3553), \mathrm{msa}=-1.8 \times 10-2$ ( the concentration of solutes $\mathrm{NaCl}$ reduces $1 \mathrm{~mol}$ $/ \mathrm{kg}$ ), $\theta \mathrm{L}=50$ 0C. If the temperature and solute molar fraction change together (chemo-thermal effect), take $\theta \mathrm{a}=\theta \mathrm{L}$. When only consider the molar fraction of solute changes (chemical effect), take $\theta \mathrm{a}=0$.

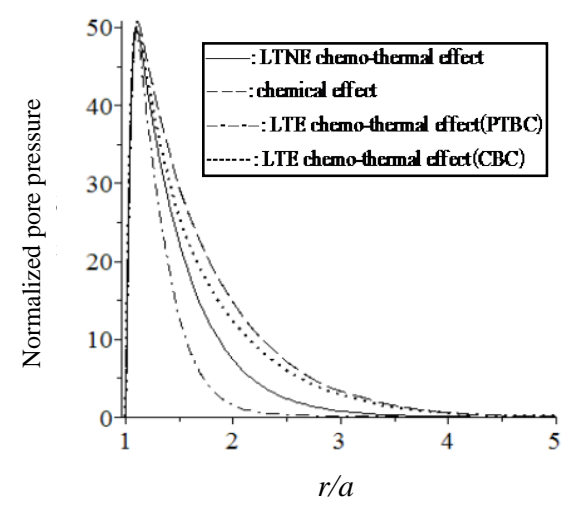

Fig. 2 Normalized pore pressure distributions along the radial direction

Fig.2 shows the normalized pore pressure distributions along the radial direction when the normalized time $\tilde{\mathrm{t}}=\mathrm{\kappa t} / \mathrm{a} 2=0.1$ and $\mathrm{Bi}=1.0$, including that under the chemical effect (only consider 
the solute molar fraction changes), the LTE and LTNE chemo- thermal effect (the solute molar fraction and temperature change together under the LTE and LTNE conditions. Fig.2 presents that the change characteristics of pore pressure along the radial direction caused by the chemical effect and the chemo- thermal effects are basically the same. The pore pressure monotonic increases rapidly along the radial distance, after reaching the peak that decreases with further increasing distance from the hole. The chemical effect is very significant to the pore pressure, and the peak of normalized pore pressure is about 50.74. Compared with the chemical effect, the influence of the changes of temperature (thermal effect) on the peak of the pore pressure is relatively weak, and the peak is about 50.0 under the LTNE and LTE chemo-thermal effects. The main difference is the speed of decay along radial distance after reaching a peak. The speed of decay under the LTE chemo-thermal effect (PTBC) is the fastest, followed by the LTNE chemo- thermal effect and the LTE chemo-thermal effect (CBC), the speed of decay under the chemical effect is the slowest. In addition, the LTNE effect in the process of decay is obvious.

Fig.3 shows the normalized radical stress distributions along the radial direction when the normalized time $\tilde{\mathrm{t}}=\kappa \mathrm{t} / \mathrm{a} 2=0.1$ and $\mathrm{Bi}=1.0$, including that under the chemical effect, the LTE and the LTNE chemo-thermal effects. Fig.3 exhibits that the variation character of radial stress along the radial direction caused by the chemical effects and the chemo-thermal effects are the same. Radial stress monotonic increases rapidly along the radial distance, after reaching the peak, and then decreases with further increasing distance from the hole. The maximum radial stress occurs in the vicinity of cavity boundary, the chemical effect is significant. The change of temperature declines the magnitude of radial stress (absolute value) in the chemo-thermal effects. The peak of normalized radial stress under the chemical effect and the LTNE and the LTE chemo-thermal effects is 27, 23, 25(CBC) and 20 (PTBC), respectively. In addition, the LTE effect is also very obvious.

Fig. 4 shows the normalized tangential stress distributions along the radial direction when the normalized time $\tilde{\mathrm{t}}=\kappa \mathrm{t} / \mathrm{a} 2=0.1$ and $\mathrm{Bi}=1.0$, including that under the chemical effect, the LTE and the LTNE chemo-thermal effects. Fig. 4 presents that the behavior of radial stress along the radial direction caused by the chemical effects and the chemo-thermal effects are similar. Near the cavity boundary, the magnitude of the tangential stress monotonically decreases with increasing distance from the hole. The tangential stresses are compressive near the cavity boundary and become tensile at larger distances from the cavity. The turn place that normalized tangential stress from compressive into tensile is about at $\mathrm{r} / \mathrm{a}=1.7$ (LTE chemo-thermal effect (PTBC)), r/a =2.2 (LTNE chemo-thermal effect) and r/a $=2.75$ (chemical effect and LTE chemo-thermal effect (CBC)), respectively. The magnitude of the compressive stress (about 75), however, is much larger than the tensile one.

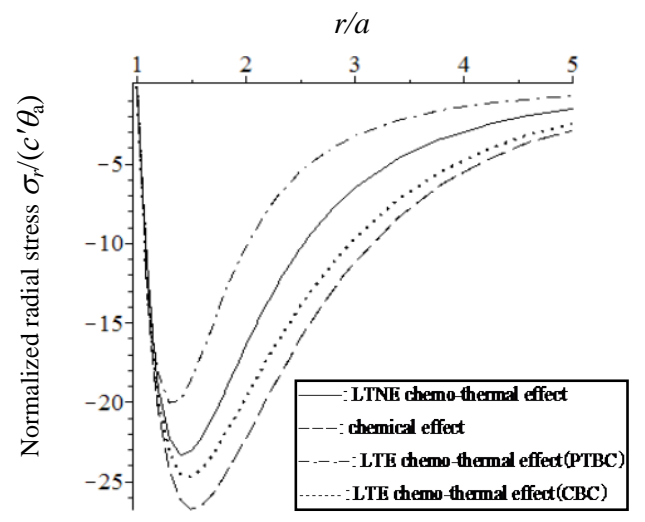

Fig.3 Normalized radial stress distributions along the radial direction 


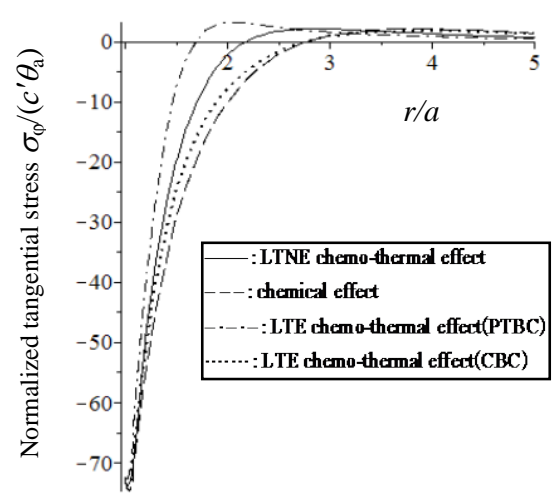

Fig.4 Normalized tangential stress distributions along the radial direction

\section{Conclusion}

In this paper, based on the chemo-thermo-poroelasticity model[12] of saturated porous medium under the LTNE conditions, the pore pressure and chemo-thermal stresses around a spherical cavity in an infinite fluid saturated porous medium subjected to sudden variations for temperature and solute mole fraction on the cavity boundary are investigated. For the non-contact stress/displacement boundary and convective boundary conditions, this problem is simplified to a spherically symmetric problem, the chemical effect, the LTE and the LTNE chemo-thermal effects are analyzed by employing Laplace transform technique and numerical method. The numerical results show that the pore pressure and stresses are dominantly affected by the chemical effect rather than thermal effect. For the radial stress, the LTNE effect is most pronounced, the change of temperature declines the magnitude of radial stress (absolute value) in the chemo-thermal effects. In addition, the pore pressure and stresses are always same order of magnitude whether considering the chemical effect or the chemo-thermal effect.

\section{References}

[1] Kurashige M. A thermoelastic theory of fluid -filled porous materials. International Journal of Solids and Structures, 25, pp.1039-1052, 1989.

[2] Kodashima T, Kurashige M. Active cooling and thermal stress reduction in a porelastic hollow sphere. Journal of Thermal Stresses, 20(3), pp.389-405,1997.

[3] Zhou Y, Rajapakse R K N D, Graham J. Coupled consolidation of a porous medium with a cylindrical or spherical cavity. Int. J. Numerical and Analytical Methods in Geo mechanics, 22, pp. 449-475, 1998.

[4] Liu Ganbin, Xie Kanghe, Zheng Rongyue. Model of nonlinear coupled thermo-hydro elastodynamics response for a saturated poro elastic medium . Science in China Series E: Technological Science, 52 (8), pp. 2373-2383, 2009.

[5] Minkowycz W J, Haji-Sheikh A and Vafai K. On the departure from local thermal equilibrium in porous media due to a rapidly changing heat source: the Sparrow number. International Journal of Heat and Mass Transfer, 42, pp. 3373-3385, 2009.

[6] He, L.-W, Jin, Z.-H. Effects of local thermal non-equilibrium on the pore pressure and thermal stresses around a spherical cavity in a porous medium. International Journal of Engineering Science, 49, pp .240-252, 2011.

[7] He L W, Jin Z H, Zhang Y. Convective cooling/heating induced thermal stresses in a fluid saturated porous medium undergoing local thermal non-equilibrium. International Journal of Solids and Structures, 49, pp .748-758, 2012.

[8] Ekbote S, Abousleiman Y. Porochemoelastic solution for an inclined boreholein in a transversely isotropic formation. Journal of Eng. Mech., 131 (5), pp. 522-533, 2005.

[9] Ekbote S, Abousleiman Y. Porochemothermo elastic solution for an inclined boreholein in a transversely isotropic formation. Journal of Eng. Mech., 132 (7), pp. 754-763, 2006.

[10]Ghassemi A, Diek A. Linear chemoporoe lasticity for swelling shales theory and application. Journal of Petroleum Science and Engineering, 38, pp. 199- 212, 2003.

[11]Ghassemi A, Diek A. Influence of coupled chemo-poro-thermoelastic processes on pore 
pressure and stress distributions around a wellbore in swelling shale. Journal of Petroleum Science and Engineering, 67, pp .57-64, 2009.

[12]Zhang Y. A Mathematical Model and Application of Saturated chemo-thermo Poroelastic Media. Shanghai: East China University of Science and Technology , 2012.

[13]Durbin F. Numerical inversion of Laplace transformation: an efficient improvement to Durbin and Abate's method. The Computer Journal, 17(4), pp.371-376, 1974. 